\title{
Utilization of educational machine tool for training of technical diagnostics based on positioning performance
}

\author{
Miroslav Cisar, ${ }^{1,}$, Ivan Kuric $^{1}$, Nadezda Cubonova ${ }^{1}$, Ivan Zajacko ${ }^{1}$, Tomas Dodok $^{1}$, \\ Anna Rudawska ${ }^{2}$ \\ ${ }^{1}$ University of Zilina, Faculty of Mechanical Engineering, Slovakia \\ ${ }^{2}$ Lublin University of Technology, Faculty of Mechanical Engineering, Lublin, Poland
}

\begin{abstract}
The technical diagnostics as essential part of machine tool maintenance provides information about condition of examined machine tool but it requires not only special equipment but also certain level of skill of operators in order to achieve optimal performance. The article is aimed at the set of methods and tools capable of providing realistic simulation of various machine tool conditions in order to allow hone skills necessary for effective diagnostician.
\end{abstract}

Keywords: Machine tool diagnostics, training, error simulation

\section{Introduction}

In order to meet customers' demands for high quality and low prices, manufacturers have to pay close attention to production resources and quality of production. The quality of machined parts is closely connected to its accuracy and by extension to quality and condition of used machine tool and its individual parts. Technical diagnostic provides various methods and tools to obtain not only current condition of machine tool but also its future progress and allows to estimate probability of fault occurrence. This allows to avoid unnecessary costs due machinery fault, unexpected downtime and production of insufficiently accurate components [1,2].

Technical diagnostics is discipline that includes wide range of methods and physical phenomena in order to detect various types of fails and changes in performance of examined device. In terms of machine tool diagnostics, there are two common elements for each of method. The first one is the machine tool on which diagnostic is performed and second one is the diagnostician. It is necessary for such person to have necessary amount of knowledge and certain level of skills in order to provide diagnostic procedures effectively and reliably. In order to keep such skills fresh or to teach new diagnostician is necessary to train certain routines. Proper training of machine tool diagnostics requires performing tasks on machines in various condition in order to gain experiences with real situations. Training of diagnostic routines in various condition requires access to machine tool, or machine

\footnotetext{
*Corresponding author: tomas.dodok@fstroj.uniza.sk

Reviewers: Andrej Czán, Alžbeta Sapietová
} 
tools, in various technical conditions. This article is aimed to simulating machine tool faults by manipulating its positioning performance $[1,3]$.

One way to achieve changes in positioning precision of machine tool is using same tools that are normally meant to reducing geometrical and positioning errors such as loosened fit in feed mechanism or using compensation tables in control system. Another approach is to change programmed tool path same way as desired error. The approach that uses modification of $\mathrm{NC}$ program can also be easily used with different devices such as industrial robots and manipulators $[4,5]$.

\section{Devices used for experiment}

Emco Concept Mill 105 (CM 105) (Fig. 1) is compact machine tool with interchangeable control system designed for industrial training and educational purposes. Such machine tools are commonly used at technical high schools and universities. The machine tool is controlled via PC with WinNC software and interchangeable control panels. In terms of training and education it is good to have one machine that can be controlled with multiple control systems but implementation of such thing reduces some functions of machine tool. For example, it is not possible to use compensation table directly in control system what reduces possibilities of training machine tool diagnostics $[6,7]$.

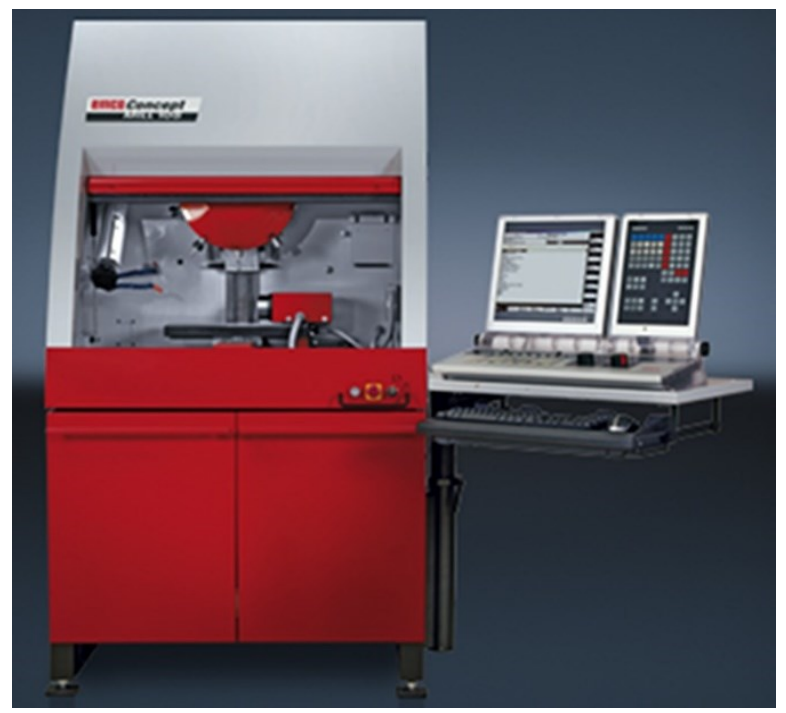

Fig. 1. EMCO Concept MILL 105 [6]

In order to confirm supposition about the possibility of simulating machine tool error by editing NC code, the Renishaw Ballbar QC20-W (Fig. 2) was selected as measuring device. Renishaw Ballbar consists of precise linear sensor, terminated by polished steel balls that fits exactly into magnetic mounts. The first ball fits to the mount held as tool in spindle; second one on pivot that is usually mounted on worktable. Unlocked mount of pivot have three degrees of freedom. That allows fast and simple aligning of mounts using setting ball in order to set center of measuring circle $[7,8,9]$.

Essentially, the Renishaw Ballbar is a linear displacement sensor designed to perform a simple, rapid check of a machine tool's positioning performance according to recognized internationals standards based on measuring deviations in the radius of circular movement measured by a transducer and captured by the computer software. The software provided with this device is able to detect and measure wide range of faults and reveal its possible 
sources. For machine tools or devices with perpendicular feeds it is possible to identify following errors and faults: backlash, cyclic error, lateral play, master-slave changeover, offset change, plot rotation, positional tolerance, radius change, reversal spikes, scaling error, servo mismatch, spiral error, squareness, slick slip, straightness, and vibration of machine. Measured data are stored in *.b5r file that is formatted similarly to XML standard which allows reading, processing, and further analysis of measured values with custom software $[9,10,11]$.

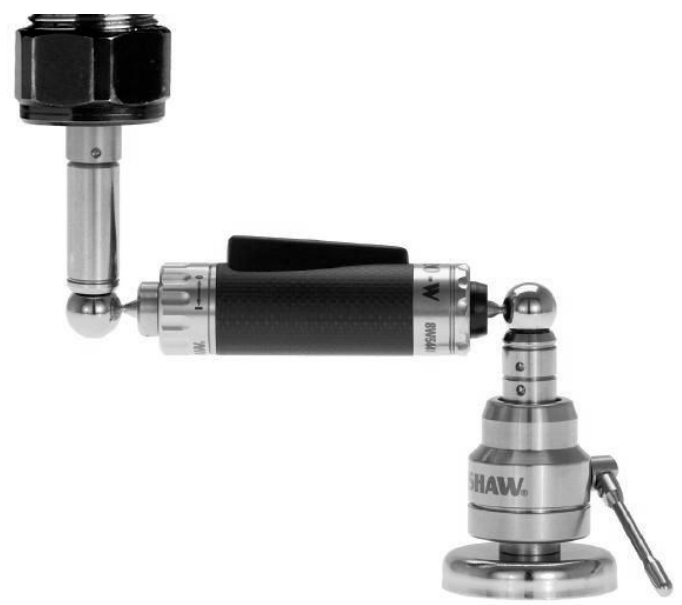

Fig. 2. Renishaw Ballbar QC20-W

\section{Simulation of machine tool geometrical errors}

As mentioned above, the CM 105 have no compensation tables in simulated control system; hence the only direct way of simulating inaccuracies and faults is actually creating them by adjusting mechanical components that are meant to correct such faults. For example it is possible to adjust position of spacer wedges in guiding (Fig. 3) and cause changes in backlash, straightness, squareness and so on.
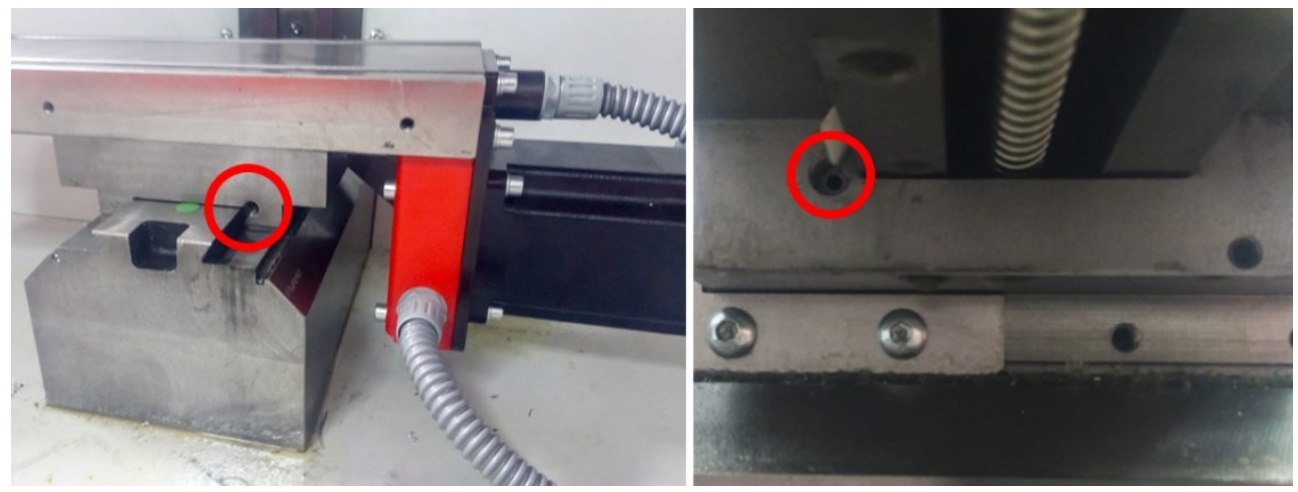

Fig. 3. Location of screws for setting position of spacer wedges on Emco Concept Mill 105

The result of measurement with loosened wedges can be additionally affected by changing of weight distribution on worktable using weights such as magnetic stands. However, this approach is relatively impractical as it requires removing casing and covers of guiding and feeds and correcting settings after measurement. 
It is not possible to easily alternate actual shape of tool path programmed thru circular interpolation and therefore circular interpolation (G02 and $\mathrm{G} 03$ ) was replaced by approximated path that consists of multiple short lines (G01). Custom software "circgen" (Fig. 4) was created at Department of Automation and Production Systems, Faculty of Mechanical Engineering, University of Zilina, in order to generate such approximated tool path. The software allows to set number of points on circle, start angle of measurement, angle of run-in and run-out path for measurement, and order of directions of measuring circles. The machine tool uses three decimal places to show coordinates, but its actual precision of positioning is far worse. Calculation of coordinates for apexes of polygon, that replaces circular path, uses radius of circumscription circle and it also includes factor of rounding coordinates to three decimal places in order to minimize deviation from radius of measured circle.

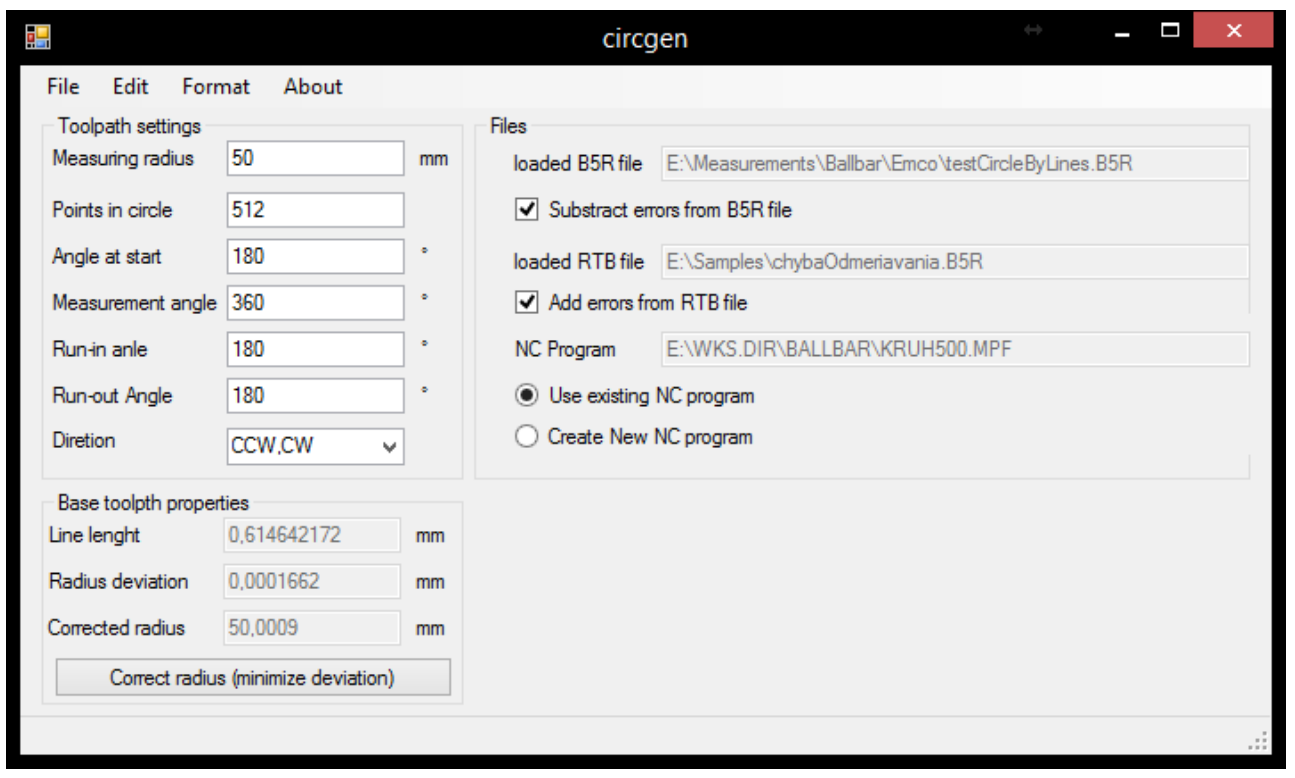

Fig. 4. User interface of the circgen software

First tests with NC program generated with circgen for control system Sinumerik 840D revealed that tool stops for moment at the end of each line that rendered measurement to be unusable. This problem was solved by adding instruction G64 (Continuous path mode). However measurement with ballbar still revealed drop in velocity. For measurement with 512 points on circle with $50 \mathrm{~mm}$ radius, the maximum velocity reached was $460 \mathrm{~mm} . \mathrm{min}-1$ regardless feedrate set in NC program. However, measurement with actual velocity of 460 $\mathrm{mm}$.min-1 is still acceptable even for measurement with velocity set to $500 \mathrm{~mm}$.min- 1 . The results of measurement with standard NC program that uses circular interpolation was essentially the same as with the NC program with polygonal path in terms of the occurrence and size of measured errors.

Next step necessary for simulation of errors is to eliminate errors that are currently occurring and can be measured. Therefore the capability of reading ballbar measurement was added to circgen software. The measured profile contains much more values than number of points used in NC program. The software uses harmonic analysis to get actual components of errors and to filter out measurement error. Main measurement error is eccentricity of measured circle and real center, it can be eliminated by setting first harmonic to zero. Then the corrected set of points is generated by calculation of deviations for each point using fast Fourier transform and recalculating $\mathrm{X}$ and $\mathrm{Y}$ coordinates for each point. 
The NC program generated with subtracted errors from previous measurement should bring measured polar diagraph closer to ideal circle but it is not possible to compensate errors in full range. Process of measuring and subtracting errors can be repeated but further subtraction have minimal to no effect as shown in Fig. 5, where the 1st iteration is the measurement that uses the uncorrected $\mathrm{NC}$ program and following iterations was measurement with path corrected according previous measurements. For comparison 0th iteration shows measurement with standard $\mathrm{NC}$ program that uses normal circular interpolation.

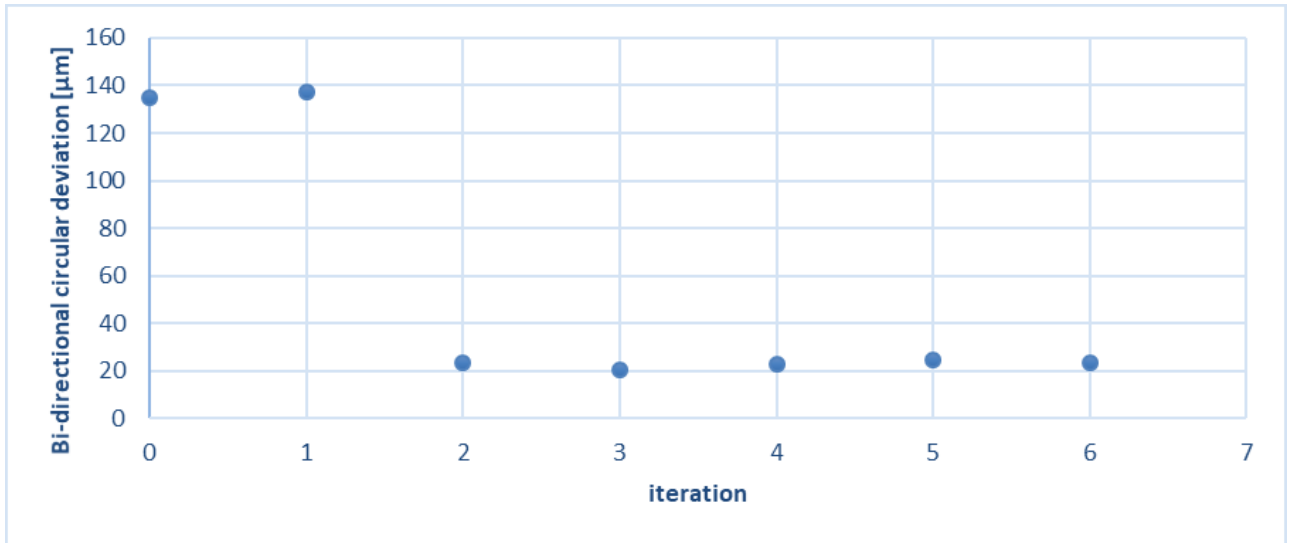

Fig. 5. Bi-directional circular deviation development after subtraction of errors measured from previous iteration

Last step for generating NC program that simulates path corresponding with some machine tool errors is adding deviations to coordinates of apexes of polygon. The source of these deviations can be the file generated in Ballbar plot simulator (Fig. 6).

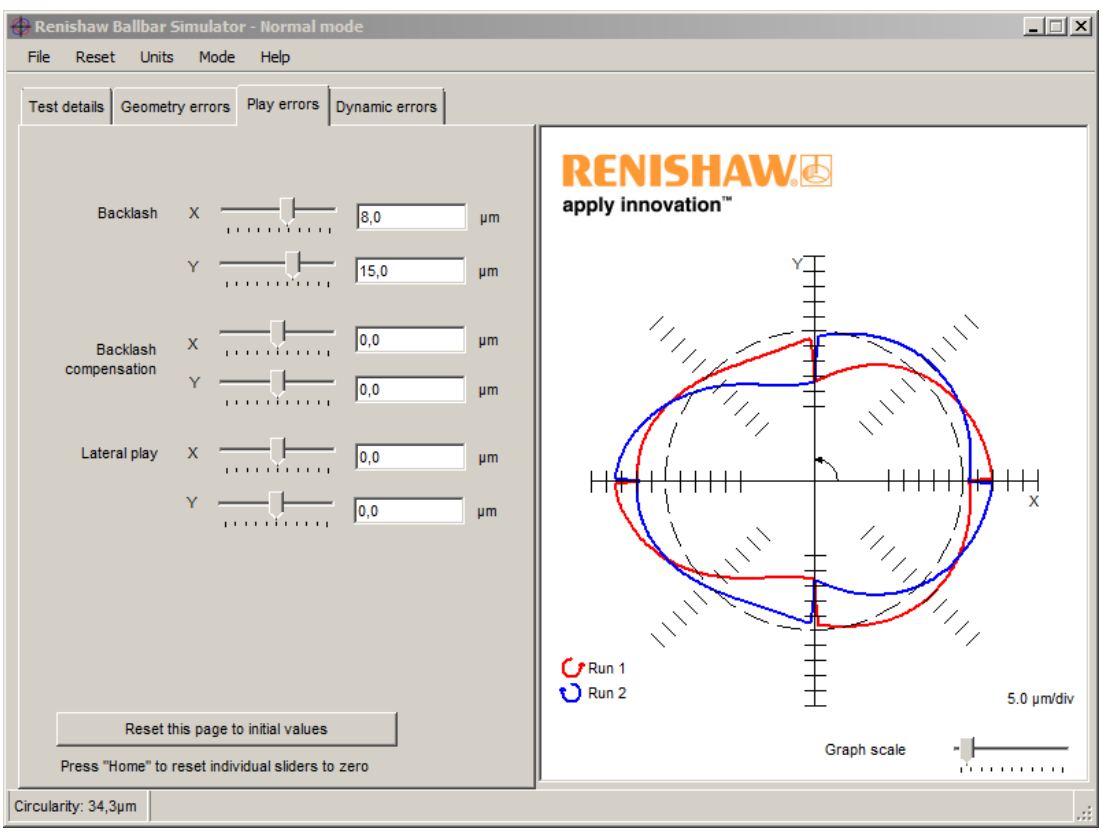

Fig. 6. Ballbar plot simulator 
This software can be used to simulate effect of various errors to shape of polar diagraph measured on ballbar. This diagraph can be saved to RTB file which contain 512 records for deviation. The count of records in RTB file was real reason to select 512 points for polygon replacing measuring circle in order to keep calculations as simple as possible.

The size of errors can be simply set by slider or by filling textboxes and saved to RTB file. Another approach to generate errors would require mathematically describe changes in circle shape caused by specific errors and adding it ideal circle to create corrected one. [9]

\section{Conclusion}

The article shows two approaches to simulating positioning errors on training machine tool Emco Concept Mill 105 and describes software tool "circgen" developed at Department of Automation and Production Systems, Faculty of Mechanical Engineering, University of Zilina. Experiments revealed that it is impossible to completely eliminate existing errors which was reduced close to $15 \%$ of original value. Such reduction is sufficient for simulating other machine tool faults without necessity of change machine tool setup mechanically. Results of measurements allows us to assume that similar technique can be applied for other methods of machine tool diagnostics involving positioning precision measurement such as laser interferometry.

This article was made under support projects KEGA 037ZU-4/2014 and KEGA 024ZU-4/2016.

\section{References}

1. M. Cisar, Diagnosis of Equipments. Mechanization and Automation Equipment for Processing, Cluj-Napoca : Publishing House Alma Mater, 209-240 (2015)

2. I. Kuric, et al., Analytical Intelligence Tools for Multicriterial Diagnostics of CNC Machines. Advances in Sci. and Tech. Res. J. 10 (32), 59-64 (2016)

3. N. Cubonova, M. Cisar, Design of Camera Mount and its Application for Monitoring Machining Process. Advances in Sci. and Tech. Res. J. 9 (26), 34-40 (2015)

4. A. Rengevic, D. Kumicakova, New Possibilities of Robot Arm Motion Simulation. Communications - Sci. Let. of the Uni. of Zilina 18 (1), 81-86 (2016)

5. D. Kumicakova et al., Specialised Robotic Hand Designing and Object Grasping Simulation. Applied Mechanics and Materials 282, 90-98 (2013)

6. Didactic Martin, s.r.o., Technical Description of Concept MILL 105 (in Slovak), Online: <www.didactic.sk/Data/802/UserFiles/emco_tech_popisy/spec_cm105-sk.pdf> (2007)

7. N. Cubonova, I. Kuric, Data Structures Implementation of the Protocol STEP-NC at CNC Machines Programming. Communications - Sci. Let. of the Uni. of Zilina 16 (3A), 176-183 (2014)

8. N. Cubonova, Computer Support Programming of Control System Sinumerik 840 D for e-learning Education. Proc. of $12^{\text {th }}$ intern. sci. conf. Engineering for Rural Development 12, 563-568 (2013)

9. Renishaw Plc., Manual: QC20-W ballbar (Czech). <http://resources.renishaw.com/ details/Manual\%3a+QC20-W+ballbar+(English)(138102)(33885)> (2011)

10. M. Saga, M. Vasko, N. Cubonova, W. Piekarska, Optimisation Algorithms in Mechanical Engineering Applications. (Pearson Education Limited, 230 p., 2015) 
11. P. Pechac, M. Saga, et al., Implementation of Memetic Algorithms into Structural Optimization. Communications Sci. Let. of the Uni. of Zilina 18 (1A), 64-69 (2016) 